\title{
Dyskeratosis Congenita
}

National Cancer Institute

\section{Source}

National Cancer Institute. Dyskeratosis Congenita. NCI Thesaurus. Code C111802.

A rare genetic disorder characterized by nail dystrophy, reticulated skin pigmentation especially on the neck and chest, and oral leukoplakia. In about half the cases mutations in the TERT, TERC, DKC1, or TINF2 genes are identified. Patients are at an increased risk of developing bone marrow failure, myelodysplastic syndrome, leukemia, or cancer, especially in the head and neck region. 